\title{
Comparison of fifteen immunoassays for the measurement of serum MUC-1/CA 15-3 in breast cancer patients
}

\author{
Marie-France Pichon ${ }^{1, *}$, Gaëlle Le Brun², Kamel \\ Hacene $^{3}$, Jean-Pierre Basuyau ${ }^{4}$, Jean-Marc \\ Riedinger ${ }^{5}$, Nicole Eche ${ }^{6}$, Yvonne Fulla $^{7}$ \\ and Natacha Charlier-Bret ${ }^{2}$ \\ ${ }^{1}$ Laboratoire d'Oncobiologie, Centre René-Huguenin, \\ Saint-Cloud, France \\ ${ }^{2}$ Agence Française de Sécurité Sanitaire des \\ Produits de Santé, Saint-Denis, France \\ ${ }^{3}$ Service de Statistiques Médicales, Centre René \\ Huguenin, Saint-Cloud, France \\ ${ }^{4}$ Laboratoire de Biologie, Centre Henri Becquerel, \\ Rouen, France \\ ${ }^{5}$ Laboratoire de Biologie Clinique, Centre Georges- \\ François Leclerc, Dijon, France \\ ${ }^{6}$ Laboratoire de Biologie Médicale, Institut Claudius \\ Regaud, Toulouse, France \\ ${ }^{7}$ Laboratoire de Radioimmunologie, Centre \\ Hospitalo-Universitaire Cochin, Paris, France
}

\begin{abstract}
Background: Quality control results for serum MUC1/CA 15-3 assays have always shown large discrepancies.

Methods: This multicentre study of 15 methods (labelled M1-M15) measured coded sera from 35 patients with breast cancer without recurrence (group 1), 46 patients at 1st metastasis (group 2), and 39 patients with advanced metastases (group 3 ). Results were compared using parametric statistics, ANOVA, principal component analysis, and receiver operating characteristic (ROC) curves.

Results: Mean MUC-1/CA 15-3 concentrations varied widely (75.1-303.0 U/mL, 24.8\%) among methods. The false positive (FP) rate for group 1 was 8/521 (1.5\%); for group 2 and group 3 false negative (FN) results were $21 / 680(3.1 \%)$ and $11 / 583(1.9 \%)$, respectively. Using the ROC cut-offs, we found no FPs for group 1 and no FNs for group 3. However, group 2 showed 16 FNs. All p-values for Pearson's correlation were $<0.0001$ between methods, except for M11. When comparing methods using different antibodies, discordance rates reached a maximum of $15.2 \%$. Principal component analysis revealed a grouping of methods using: CanAg monoclonal antibodies (mAbs) (M2, M7 and M12); Centocor/Fujirebio mAbs (M3-M6, M8-M10, M14-M15) and Biomira mAbs (M1 and M13); and Centocor/Fujirebio mAbs (M11).
\end{abstract}

*Corresponding author: Dr. Marie-France Pichon, Laboratoire d'Oncobiologie, Centre René-Huguenin, Saint-Cloud, France

Phone: +33 (6) 295320 95, E-mail: labonco@crh1.org Received February 12, 2009; accepted April 30, 2009; previously published online June 24, 2009
Conclusions: Results were more consistent among methods using the same antibody type. Principal component analysis showed that antibody type was the strongest determinant of immunoassay results. Clin Chem Lab Med 2009;47:985-92.

Keywords: cancer antigen (CA) 15-3 immunoassays; tumour markers.

\section{Introduction}

Tumour marker cancer antigen (CA) 15-3 and its analogues, defined as MUC-1/CA 15-3, correspond to an immunodominant epitope of the protein core of the membrane MUC-1 mucin, a highly heterogeneous molecule. Located in the extracellular domain of the MUC-1 mucin, this epitope resides within a hydrophilic region of seven amino acids (PDTRPAP) belonging to the 20 amino acids tandem repeat sequences. MUC-1 mucin is overexpressed in carcinomas $(1,2)$. Increased concentrations of MUC-1/CA 15-3 are found in metastatic breast cancer and serial assays of this marker are used to monitor tumour response during treatment (3).

The CA 15-3 tumour marker is not a clearly defined analyte. The original CA 15-3 assay is defined by two monoclonal antibodies (mAbs): 115 D8, developed against milk fat globule, and DF3, developed against membranes of human breast cancer cells (Centocor) Fujirebio). The DF3 mAb recognises the epitope APDTRPAP, a sequence that contains the common DTR motif recognised by more than 20 different antiMUC-1 antibodies (4-8). These two mAbs are components of several MUC-1/CA 15-3 immunoassay formats used in clinical settings. Other mAbs that recognise amino acid sequences spanning the DTR motif, such as B27.29 (PDTRPAP) (Biomira) or Ma 552 (PDTRPAPG) (CanAg), are also used for measurement of MUC-1/CA 15-3 in breast cancer (9).

Method comparison studies and inter-laboratory quality control for serum MUC-1/CA 15-3 assays have always shown large discrepancies among results (10-12). For that reason, the French Health Products Agency, Agence Française de Sécurité Sanitaire des Produits de Santé (AFSSAPS) launched a multicentre evaluation of 15 methods marketed in France for the measurement of CA 15-3 or of CA 15-3 analogs, CA 27.29, BR, or BR-MA (CA 15-3). To facilitate comparisons among methods, all 12 participating laboratories tested the same serum samples. Results of the AFSSAPS evaluation showing the clinical sensitivity and specificity of the different methods have been published (13). The present work focuses on the statistical analysis of the AFSSAPS panel data, in order to help define the origin of discrepancies in MUC-1/ 
CA 15-3 concentrations when using different methods on the same samples.

\section{Patients and methods}

\section{Patients and sera}

For serum MUC-1/CA 15-3 assays, there is no primary reference material for calibration or a reference method for comparison. Therefore, we used the clinical status of a panel of breast cancer patients to compare the performance of the different methods in terms of clinically adequate MUC-1/CA 15-3 results. We established a protocol that defined three patient groups. We excluded, as best as possible, risks of both false negative (FN) MUC-1/CA 15-3 results and non-specific increases in MUC-1/CA 15-3. The selection of sera to be included in the panel and distributed to participating laboratories was based on strictly defined clinical criteria approved by three expert oncologists.

At the time of blood sampling, clinical status of patients was evaluated by oncological examination and medical imaging, pathology, and laboratory tests, according to the Standards Options and Recommendations of the French Fédération des Centres de Lutte Contre le Cancer (14). All cancer related events during patient follow up had to be documented by at least one imaging technique (X-rays, CT-scan, echography, PET-scan) or by puncture cytology, biopsies, or pathologic examination following surgical removal.

Group 1, comprised of breast cancer patients undergoing active follow-up following primary treatment, showed no recurrence during an observation period of at least 4 years after initial diagnosis. Group 2 consisted of patients at their first metastasis, with blood collection performed between 0 and 90 days before proven metastasis in order to take into account an eventual lead time phenomenon. Group 3 patients were all known to have metastases, with blood collection performed at least 90 days after the diagnosis of metastases. Five exclusion rules, based on case types, were strictly followed: all cases with oestrogen receptor-negative tumours, since the $M U C-1$ gene is regulated by oestrogens; cases with increased MUC-1/CA 15-3 without any proven recurrence; cases with proven recurrence without increases in MUC-1/CA 15-3; cases with isolated local recurrences or recurrences preceding a first metastasis; and cases with previous or associated second cancer. Additional exclusion criteria were defined as: pregnancy, associated breast benign pathologies, associated inflammatory, infectious or autoimmune diseases, patients with significant metabolic alterations.

Frozen $\left(\leq-20^{\circ} \mathrm{C}\right)$ serum samples were retrieved from blood banks (MFP, JPB, NE, and JMR), or obtained from patients at their respective Cancer Centres. A minimum of $2 \mathrm{~mL}$ of serum was required for analysis by all methods. Initial CA 15-3 concentration was measured by immunoradiometric assay (ELSA CA 15-3, Cis Bio International, Gif sur Yvette, France, MFP) or by immunofluorometric assay (Kryptor, Brahms, Clichy, France, JPB, NE, JMR). This study was performed in accordance with the Declaration of Helsinki Ethical Guidelines.

\section{Methods evaluated}

This study tested 15 assay methods, both manual and automated, available in the French market in 2005: Architect CA 15-3 and Axsym CA 15-3 (Abbott Diagnostic, Rungis, France), CA 15-3 Kryptor (Brahms, Clichy, France), Advia Centaur CA 15-3 and Advia Centaur BR CA 15-3 (Bayer Diagnostics,
Puteaux, France), Access BR monitor (Beckman Coulter, Villepinte, France), Vidas CA 15-3 (Biomerieux, Marcy I'Etoile, France), ELSA CA 15-3 (Cis Bio International, Gif sur Yvette, France), Liaison CA 15-3 and CA 15-3 IRMA (DIA Sorin, Antony, France), Immulite 2000/2500 BR-MA (DPC, La Garenne-Colombes, France), IRMA MUC-1 CA 15-3 (Immunotech, Marseille, France), Vitros CA 15-3 (Ortho Clinical Diagnostics, Issy-les Moulineaux, France), Elecsys CA 15-3 II (Roche Diagnosis, Meylan, France), and AIA Pack 27.29, (Tosoh Bioscience, Saran, France). When identical assay methods were available for use with different immunoassay systems, only the most popular was evaluated. Immunoradiometric assays, Bayer Advia Centaur, and Brahms Kryptor methods were evaluated in AFSSAPS laboratories (MFP, JPB, JMR, NE, YF). For the other methods, the choice of the laboratories in charge of the assays was made in concert with the manufacturers. All biologists had to follow manufacturer's recommendations exactly. Serum samples were coded before being sent to the participating laboratories for double blind assays to eliminate any indication of patient groups.

\section{Statistical methods}

Our statistical comparisons of methods did not involve the use of any particular method as a reference method. MUC1/CA 15-3 concentrations were analysed as continuous or dichotomised (normal/increased) variables, according to the cut-off values given by each manufacturer. For the transformation of concentrations close to cut-off levels into binary variables, we considered non-significant differences to be those values within $5 \%$ of the cut-off level.

All statistical analyses were performed using SAS Statistical Software packages (version 8.2, SAS Institute, Cary, NC, USA). Descriptive statistics have been computed for all the measurements in the overall group, as well as in the three defined patient subgroups. Relationships between different methods were analysed using a parametric test, with determination of Pearson's correlation coefficient. Agreement between methods was calculated using the intraclass correlation coefficient (ICC). A multivariate variance analysis (MANOVA) was performed in order to assess the group effect taking into account all methods. Factor analysis began with a matrix of intercorrelations among all methods used in the evaluation. The goal of factor analysis is to determine a smaller number of factors that help explain these correlations. Prior to submitting data for analysis, they were inspected for meeting the assumptions of factor analysis. The suitability assessment of the correlation matrix for factor analysis involved the computation of the Kaiser-Meyer-Olkin index, the Bartlett's test of sphericity and the examination of the anti-image covariance matrix. Once the correlation matrix has been determined as suitable for factor analysis, the chosen method for factor extraction was the principal components analysis (PCA) with the Varimax rotation for improving interpretability of retained factors. These retained factors have been determined by the Scree test as well as by the Kaiser rule.

Receiver operating characteristic analysis $(\mathrm{ROC})$ was performed using MedCalc statistical software (v. 9.3, Mariakerke, Belgium).

\section{Results}

Sera from 35 patients in group 1, 46 patients in group 2 , and 39 patients in group 3 fulfilled the requirements for inclusion in the panel to be analysed. The mean 
age of patients was $54.9 \pm 12.8$ years, and $72.8 \%$ were menopausal. Methods were randomly labelled M1-M15. One hundred and twenty serum samples were assayed using 15 methods; 10 using Centocor/ Fujirebio mAbs (M3-6, M8-11, M14-15), 3 using CanAg mAbs (M2, 7, 12) and 2 using B 27.29 (Biomira) mAbs (M1 et M13). Results from method M8 were asked to be withdrawn by the manufacturer because of possible underestimation with the current reagent. However, since no significant difference was found between these results and those obtained with the other methods, we decided to keep results from M8 in the statistical analyses.

A total of 1792 MUC-1/CA 15-3 results were obtained. Eight were missing because of insufficient serum volume for some assays. One thousand seven hundred and sixteen results were reported as continuous variables and were dichotomised; 76 results were used as dichotomous variables since six were below the detection limits of the assay; and 70 were greater than the highest concentration used for the standard curve, and with insufficient serum volume for reanalysis.

\section{Stability of CA $15-3$ in deep frozen serum samples}

We verified that CA $15-3$ concentrations were not altered after the process of thawing, aliquoting and refreezing required to provide samples to all participating laboratories. Forty-eight blood bank sera, initially assayed by ELSA CA 15-3, were further tested in a different laboratory using the same method. Results correlated well: the regression line obtained was y (2nd assay $)=-0.1394+0.9756 \times(1$ st assay), $\mathrm{p}<0.0001$, with mean \pm SD CA $15-3$ concentrations of $51.8 \pm 47.8 \mathrm{U} / \mathrm{mL}$ at 1 st assay vs. $50.4 \pm 47.2 \mathrm{U} / \mathrm{mL}$ after retesting (non-significant difference). The same comparison was done for the Kryptor ${ }^{\circledR}$ technique and a good correlation was also observed: regression equation was y (2nd assay $)=1.8234+0.9311 \times(1$ st assay), $\mathrm{p}<0.0001$, with mean \pm SD initial CA $15-3$ of $108.1 \pm$ $91.8 \mathrm{U} / \mathrm{mL}$ vs. $102.4 \pm 87.5 \mathrm{U} / \mathrm{mL}$ after dispatching ( $n=62$, non-significant difference). Thus, despite a slight decrease after producing aliquots for analysis, we considered that MUC-1/CA 15-3 was not significantly affected by freezing and thawing.

\section{Descriptive statistics}

Tables 1-4 show the distribution of MUC-1/CA 15-3 concentrations according to the different methods and patient groups. Depending on the method, mean MUC-1/CA 15-3 concentrations vary considerably (75.1-303.0 U/mL, 24.8\%, Table 1). For each method, a variance analysis of the MUC-1/CA 15-3 results by patient groups showed a significant difference in all cases $(p<0.0001)$, thus validating the choice of serum for each group.

Variation between different methods (and different antibodies) was more obvious in sera from patients with recurrence compared to patients from group 1 who were expected to have low MUC-1/CA 15-3 concentrations. In group 1, two methods (M1, $\mathrm{n}=7, \mathrm{M} 4$, $\mathrm{n}=1$ ) gave false positive (FP) (increased) results, corresponding to an overall FP rate of $8 / 521$ (1.5\%). For group 2, six methods (M3, $n=2, M 7, n=6, M 8, n=3$, $M 10, n=2, M 11, n=6, M 14, n=2)$ gave FN (normal) results, with a total of $21 / 680$ (3.1\%) FN results. Group 3 showed 11/583 (1.9\%) FN results: M1, M2, M3, M12, M13 and M14 methods each showed 1 FN result, M7 and M8 methods 3 and 2 FN results, respectively.

\section{Correlations}

Within the overall patient group, and between methods taken two by two, all p-values for Pearson's correlation coefficients were $<0.0001$, except for method M11. For the latter, all Pearson's correlation coefficients were $<0.60$ (non-significant) with regard to methods M3 $(n=107, p=0.1962), M 5(n=107, p=$ 0.0968), M7 ( $n=105, \quad p=0.1565), \quad M 13 \quad(n=107$, $p=0.1520), M 14(n=107, p=0.1484)$ and M15 $(n=107$, $p=0.1556)$. When calculating pairwise ICCs by ANOVA to evaluate homogeneity between results obtained by the different methods, results from group 1 patients were found to be more homogeneous than those from other groups (Table 5). ICC results grouped by antibody type showed different levels of

Table 1 All patients. Distribution of MUC-1/CA 15-3 concentrations $(\mathrm{U} / \mathrm{mL})$ according to the different methods.

\begin{tabular}{|c|c|c|c|c|c|c|c|c|c|}
\hline Method & $\mathrm{mAb}$ & $\mathrm{n}$ & Mean & SD & Median & Minimum & Maximum & 1st quartile & 3rd quartile \\
\hline M1 & Biomira & 113 & 92.4 & 92.6 & 58.7 & 6.2 & 395.0 & 24.6 & 125.5 \\
\hline M13 & Biomira & 119 & 303.0 & 1683.5 & 81.1 & 4.0 & $18,315.2$ & 24.3 & 190.4 \\
\hline M2 & CanAg & 119 & 176.1 & 281.7 & 90.0 & 8.0 & 1719.0 & 32.0 & 184.0 \\
\hline M7 & CanAg & 118 & 165.8 & 859.0 & 42.2 & 4.0 & 9301.0 & 18.3 & 88.4 \\
\hline M12 & CanAg & 103 & 75.2 & 60.1 & 63.1 & 7.9 & 247.0 & 20.7 & 118.4 \\
\hline M3 & Cen/Fuji & 120 & 276.5 & 1734.3 & 54.4 & 4.4 & $18,983.0$ & 21.1 & 145.4 \\
\hline M4 & Cen/Fuji & 118 & 129.3 & 186.3 & 56.0 & 5.6 & 1025.0 & 23.3 & 162.7 \\
\hline M5 & Cen/Fuji & 120 & 202.8 & 911.2 & 61.0 & 6.0 & 9827.0 & 20.5 & 144.0 \\
\hline M6 & Cen/Fuji & 114 & 98.5 & 115.0 & 55.4 & 5.3 & 628.0 & 21.4 & 139.3 \\
\hline M8 & Cen/Fuji & 111 & 75.1 & 75.1 & 45.1 & 5.4 & 337.0 & 19.3 & 117.1 \\
\hline M9 & Cen/Fuji & 111 & 110.2 & 114.5 & 66.3 & 6.6 & 488.0 & 24.8 & 159.0 \\
\hline M10 & Cen/Fuji & 104 & 64.3 & 59.0 & 41.6 & 7.3 & 240.1 & 18.5 & 91.1 \\
\hline M11 & Cen/Fuji & 107 & 60.7 & 57.6 & 39.7 & 2.6 & 275.8 & 18.6 & 86.1 \\
\hline M14 & Cen/Fuji & 120 & 229.2 & 1210.5 & 54.0 & 2.7 & $13,183.6$ & 20.6 & 147.0 \\
\hline M15 & Cen/Fuji & 120 & 265.2 & 1454.0 & 63.7 & 4.9 & $15,839.2$ & 22.9 & 143.6 \\
\hline
\end{tabular}

mAb, monoclonal antibodies; Cen/Fuji, Centocor/Fujirebio. 
Table 2 Group 1 (patients without recurrence). Distribution of MUC-1/CA 15-3 concentrations (U/mL) according to the different methods.

\begin{tabular}{|c|c|c|c|c|c|c|c|c|c|}
\hline Method & $\mathrm{mAb}$ & $\mathrm{n}$ & Mean & SD & Median & Minimum & Maximum & 1st quartile & 3rd quartile \\
\hline M1 & Biomira & 35 & 16.2 & 7.1 & 14.8 & 6.2 & 30.9 & 10.0 & 22.2 \\
\hline M13 & Biomira & 34 & 14.7 & 7.2 & 13.3 & 4.0 & 30.2 & 9.6 & 19.9 \\
\hline M2 & CanAg & 35 & 20.9 & 9.0 & 18.0 & 8.0 & 39.0 & 13.0 & 28.0 \\
\hline M7 & CanAg & 33 & 12.0 & 6.0 & 9.9 & 4.0 & 25.6 & 7.3 & 15.2 \\
\hline M12 & CanAg & 35 & 17.1 & 6.2 & 16.1 & 7.9 & 34.3 & 10.1 & 16.1 \\
\hline M3 & Cen/Fuji & 35 & 13.5 & 5.7 & 12.6 & 4.4 & 24.7 & 8.7 & 19.3 \\
\hline M4 & Cen/Fuji & 35 & 15.5 & 6.3 & 14.0 & 5.6 & 28.9 & 9.4 & 21.6 \\
\hline M5 & Cen/Fuji & 35 & 14.0 & 4.9 & 13.0 & 6.0 & 23.0 & 10.0 & 18.0 \\
\hline M6 & Cen/Fuji & 35 & 15.4 & 5.8 & 14.8 & 5.3 & 25.1 & 10.8 & 21.0 \\
\hline M8 & Cen/Fuji & 35 & 13.6 & 5.9 & 13.1 & 5.4 & 24.7 & 7.7 & 18.0 \\
\hline M9 & Cen/Fuji & 35 & 17.6 & 7.6 & 15.5 & 6.6 & 31.5 & 11.0 & 24.7 \\
\hline M10 & Cen/Fuji & 35 & 14.3 & 4.7 & 14.0 & 7.3 & 25.0 & 9.9 & 18.8 \\
\hline M11 & Cen/Fuji & 31 & 12.8 & 6.4 & 11.9 & 2.6 & 24.9 & 7.4 & 18.0 \\
\hline M14 & Cen/Fuji & 35 & 12.2 & 6.1 & 10.5 & 2.7 & 22.9 & 8.2 & 17.8 \\
\hline M15 & Cen/Fuji & 35 & 14.5 & 6.1 & 12.7 & 4.9 & 26.9 & 9.4 & 19.7 \\
\hline
\end{tabular}

mAb, monoclonal antibodies; Cen/Fuji, Centocor/Fujirebio.

Table 3 Group 2 (patients at first metastasis). Distribution of MUC-1/CA 15-3 concentrations (U/mL) according to the different methods.

\begin{tabular}{|c|c|c|c|c|c|c|c|c|c|}
\hline Method & $\mathrm{mAb}$ & $\mathrm{n}$ & Mean & SD & Median & Minimum & Maximum & 1st quartile & 3rd quartile \\
\hline M1 & Biomira & 44 & 88.0 & 68.2 & 71.7 & 28.8 & 382.2 & 50.2 & 92.9 \\
\hline M13 & Biomira & 46 & 512.6 & 2686.0 & 81.5 & 46.1 & $18,315.2$ & 60.8 & 106.7 \\
\hline M2 & CanAg & 45 & 170.5 & 280.1 & 98.0 & 46.0 & 1719.0 & 63.0 & 134.0 \\
\hline M7 & CanAg & 46 & 284.8 & 1365.7 & 46.9 & 19.3 & 9301.0 & 34.3 & 72.3 \\
\hline M12 & CanAg & 42 & 85.2 & 43.0 & 73.2 & 40.5 & 238.7 & 55.1 & 93.2 \\
\hline M3 & Cen/Fuji & 46 & 487.9 & 2788.3 & 55.4 & 28.3 & $18,983.0$ & 42.1 & 87.6 \\
\hline M4 & Cen/Fuji & 45 & 90.3 & 92.1 & 58.3 & 24.6 & 461.0 & 43.5 & 97.8 \\
\hline M5 & Cen/Fuji & 46 & 298.4 & 1438.7 & 61.0 & 30.0 & 9827.0 & 44.0 & 88.0 \\
\hline M6 & Cen/Fuji & 44 & 81.9 & 66.0 & 57.8 & 29.8 & 332.8 & 48.2 & 83.1 \\
\hline M8 & Cen/Fuji & 43 & 68.4 & 54.9 & 52.6 & 21.3 & 337.0 & 39.5 & 76.5 \\
\hline M9 & Cen/Fuji & 43 & 105.5 & 94.7 & 75.3 & 33.5 & 456.0 & 57.4 & 108.0 \\
\hline M10 & Cen/Fuji & 43 & 63.2 & 36.6 & 53.9 & 22.6 & 185.1 & 38.0 & 84.0 \\
\hline M11 & Cen/Fuji & 46 & 59.7 & 46.9 & 40.9 & 13.0 & 252.6 & 32.7 & 65.2 \\
\hline M14 & Cen/Fuji & 46 & 363.4 & 1933.3 & 55.2 & 22.8 & $13,183.6$ & 40.6 & 94.3 \\
\hline M15 & Cen/Fuji & 46 & 430.2 & 2324.2 & 63.9 & 36.3 & $15,839.2$ & 46.9 & 83.6 \\
\hline
\end{tabular}

mAb, monoclonal antibodies; Cen/Fuji, Centocor/Fujirebio.

Table 4 Group 3 (patients with metastasis). Distribution of MUC-1/CA 15-3 concentrations (U/mL) according to the different methods.

\begin{tabular}{|c|c|c|c|c|c|c|c|c|c|}
\hline Method & $\mathrm{mAb}$ & $\mathrm{n}$ & Mean & SD & Median & Minimum & Maximum & 1st quartile & 3rd quartile \\
\hline M1 & Biomira & 34 & 176.6 & 97.5 & 155.9 & 32.90 & 395.0 & 114.5 & 215.8 \\
\hline M13 & Biomira & 39 & 307.1 & 359.1 & 209.6 & 35.30 & 2140.3 & 147.3 & 338.8 \\
\hline M2 & CanAg & 39 & 321.7 & 333.8 & 198.0 & 32.00 & 1586.0 & 126.0 & 388.0 \\
\hline M7 & CanAg & 39 & 155.7 & 159.7 & 88.4 & 18.10 & 673.9 & 62.3 & 215.8 \\
\hline M12 & CanAg & 26 & 137.3 & 52.5 & 144.2 & 30.90 & 247.0 & 99.7 & 165.0 \\
\hline M3 & Cen/Fuji & 39 & 263.3 & 308.5 & 166.3 & 28.20 & 1676.0 & 110.2 & 265.7 \\
\hline M4 & Cen/Fuji & 38 & 280.3 & 249.2 & 193.1 & 38.53 & 1025.0 & 127.7 & 352.1 \\
\hline M5 & Cen/Fuji & 39 & 259.9 & 319.2 & 152.0 & 29.00 & 1911.0 & 125.0 & 275.0 \\
\hline M6 & Cen/Fuji & 35 & 202.6 & 140.7 & 169.0 & 31.10 & 628.0 & 103.8 & 169.0 \\
\hline M8 & Cen/Fuji & 33 & 149.1 & 74.4 & 135.2 & 19.54 & 320.1 & 107.7 & 178.6 \\
\hline M9 & Cen/Fuji & 33 & 214.7 & 112.1 & 207.0 & 41.60 & 488.0 & 138.0 & 266.0 \\
\hline M10 & Cen/Fuji & 26 & 133.5 & 60.1 & 130.6 & 33.10 & 240.1 & 93.7 & 179.1 \\
\hline M11 & Cen/Fuji & 30 & 111.6 & 59.6 & 106.2 & 30.40 & 275.8 & 64.9 & 149.0 \\
\hline M14 & Cen/Fuji & 39 & 265.8 & 310.0 & 170.0 & 28.30 & 1691.6 & 122.5 & 253.0 \\
\hline M15 & Cen/Fuji & 39 & 295.7 & 363.0 & 165.7 & 31.70 & 1898.5 & 127.1 & 296.9 \\
\hline
\end{tabular}

mAb, monoclonal antibodies; Cen/Fuji, Centocor/Fujirebio. 
homogeneity: with B 27.29 (Biomira, 2 methods), all ICCs were $>0.75$, for CanAg antibodies (3 methods), the majority of ICCs were between 0.40 and 0.75 and with Centocor/Fujirebio antibodies (10 methods), the majority of ICCs was $>0.75$ (Table 6).

\section{Analysis of discordances between methods}

In this analysis, MUC-1/CA 15-3 concentrations were analysed as binary variables. Table 7 shows the percent discordance by methods taken two by two in the overall patient group. Maximum discordance was $7.5 \%$ for methods using Biomira (CA 27.29) mAbs, $8.5 \%$ for CanAg mAbs and $5.9 \%$ for Centocor/Fujirebio mAbs. When comparing methods using different antibodies, discordance rates were found to be greater (up to $15.2 \%$ ).

Manufacturers' calculations for cut-off values were heterogeneous and consequently could impact classification of patients. To verify if the cut-off values provided by the manufacturers were optimised, we performed ROC curve analysis using group 1 patients as non-recurring and groups 2 and 3 as patients with recurrence. For two methods only, the cut-off values obtained using ROC analysis differed by $<10 \%$ from the one given in the manufacturer's instructions (Table 8). When using the ROC cut-offs to calculate the sensitivity and specificity of each method, no FP results for group 1 patients and no $\mathrm{FN}$ results for group 3 patients were obtained. For patients in group $2,16 \mathrm{FN}$ results were obtained $(n=1$ for methods $M 1$, $M 2, M 4, M 10$ and $M 12, n=3$ for $M 8$, and $n=4$ for $M 7$ and $\mathrm{M} 11$ ).

\section{Principal component analysis}

To explore the interrelationships among methods, all 15 methods were subjected to PCA for the total group.
Prior to performing PCA, the suitability of the data for factor analysis was assessed. Inspection of the correlation matrix revealed the presence of many coefficients above 0.3. The Kaiser-Meyer-Olkin index was 0.92 , exceeding the recommended value of 0.6 and Barlett's test of sphericity reached statistical significance $(p<0.00001)$, supporting factoring of the correlation matrix. The Kaiser rule (eigenvalues $>1$ ) and the Scree plot of eigenvalues showed that the 15 variables corresponding to the methods could be reduced to two factors, explaining $96.4 \%$ of the variance in the overall patient group. Final results after Varimax rotation are shown in Figure 1, where the influence of the antibody type is indicated by the visual grouping of methods. Three groups of methods can be discerned: M2, M7 and M12 using CanAg antibodies, a large group including $\mathrm{M} 1$ and $\mathrm{M} 13$ (Biomira mAbs) together with M3-M6, M8-M10, M14-M15 (Centocor/Fujirebio mAbs), and M11, using Centocor/Fujirebio $\mathrm{mAbs}$, was isolated in a third group. Among IRMA assays (M10, M11 and M12), M12 using CanAg $m A b s$ is in the area of the other methods using CanAg mAbs, but not in the Centocor/Fujirebio mAbs groups.

\section{Discussion}

Most molecules used as tumour markers are derived from complex glycoproteins. Serum markers such as MUC-1/CA 15-3 have an abnormal structure in cancer cells, and their molecular structures vary according to tumour type (15). With the exception of $\alpha$-fetoprotein, human choriogonadotrophin, and prostate specific antigen, such molecular complexity has hampered the preparation of reference material and the development of an internationally accepted reference method $(16,17)$. This may help explain the challenge

Table 5 Results of ANOVA: pairwise intraclass correlation coefficient (ICC).

\begin{tabular}{|c|c|c|c|c|}
\hline ICC & Group 1 & Group 2 & Group 3 & All patients \\
\hline $\mathrm{ICC} \leq 0.40$ & $1(1 \%)$ & $15(14 \%)$ & $11(10 \%)$ & $8(8 \%)$ \\
\hline $0.40<\mathrm{ICC} \leq 0.75$ & $32(30 \%)$ & 37 (35\%) & $44(42 \%)$ & $18(17 \%)$ \\
\hline $0.75<\mathrm{ICC} \leq 0.90$ & $41(39 \%)$ & $38(36 \%)$ & $31(30 \%)$ & $44(42 \%)$ \\
\hline$I C C>0.90$ & $31(30 \%)$ & $15(14 \%)$ & $19(18 \%)$ & $35(33 \%)$ \\
\hline Total ICC $\geq 0.75$ & 72 (69\%) & $53(50 \%)$ & $50(48 \%)$ & $79(75 \%)$ \\
\hline
\end{tabular}

Table 6 Pairwise intraclass correlation coefficient (ICC) according to the type of antibody (ANOVA).

\begin{tabular}{|c|c|c|c|c|c|c|}
\hline Antidody & Method & Group & $\mathrm{ICC} \leq 0.40$ & $0.40<\mathrm{ICC} \leq 0.75$ & $0.75<\mathrm{ICC} \leq 0.90$ & ICC $>0.90$ \\
\hline Biomira & M1, M13 & 1 & 0 & 0 & 0 & $1(100 \%)$ \\
\hline \multirow[t]{3}{*}{ (CA 27.29) } & & 2 & 0 & 0 & $1(100 \%)$ & 0 \\
\hline & & 3 & 0 & 0 & $1(100 \%)$ & 0 \\
\hline & & All patients & 0 & 0 & $1(100 \%)$ & 0 \\
\hline \multirow[t]{4}{*}{ CanAg } & M2, M7, M12 & 1 & 0 & $2(67 \%)$ & $1(33 \%)$ & 0 \\
\hline & & 2 & 0 & $3(100 \%)$ & 0 & 0 \\
\hline & & 3 & $1(33 \%)$ & $2(67 \%)$ & 0 & 0 \\
\hline & & All patients & 0 & $2(67 \%)$ & $1(33 \%)$ & 0 \\
\hline Centocor/ & M3-M6, & 1 & $0(0 \%)$ & $8(18 \%)$ & $15(33 \%)$ & $22(49 \%)$ \\
\hline \multirow[t]{3}{*}{ Fujirebio } & M8-M11, & 2 & $4(9 \%)$ & $9(20 \%)$ & $23(51 \%)$ & $9(20 \%)$ \\
\hline & M14, M15 & 3 & $1(2 \%)$ & 15 (33\%) & $17(38 \%)$ & $12(27 \%)$ \\
\hline & & All patients & $4(9 \%)$ & $2(4 \%)$ & $16(36 \%)$ & $23(51 \%)$ \\
\hline
\end{tabular}

Group 1, patients without recurrance; group 2, patients at their 1st metastasis; group 3, patients with advanced metastasis. 
Table 7 All patients. Proportion of discordant binary results by methods.

\begin{tabular}{|c|c|c|c|c|c|c|c|c|c|c|c|c|c|c|c|}
\hline & M1* & M13* & $\mathrm{M} 2 * *$ & M7** & M12** & M3 & M4 & M5 & M6 & M8 & M9 & M10 & M11 & M14 & M15 \\
\hline M1* & 0.0000 & 0.0750 & 0.0667 & 0.1525 & 0.0667 & 0.0833 & 0.0583 & 0.0750 & 0.0678 & 0.1176 & 0.0678 & 0.0833 & 0.1083 & 0.0833 & 0.0583 \\
\hline M13* & & 0.0000 & 0.0083 & 0.0763 & 0.0083 & 0.0250 & 0.0333 & 0.0167 & 0.0254 & 0.0059 & 0.0254 & 0.0417 & 0.0667 & 0.0250 & 0.0167 \\
\hline M2** & & & 0.0000 & 0.0847 & 0.0167 & 0.0333 & 0.0417 & 0.0250 & 0.0339 & 0.0672 & 0.0339 & 0.0500 & 0.0750 & 0.0333 & 0.0250 \\
\hline $\mathrm{M} 7 * *$ & & & & 0.0000 & 0.0847 & 0.0847 & 0.1102 & 0.0932 & 0.0862 & 0.0940 & 0.1034 & 0.1186 & 0.1271 & 0.0847 & 0.0932 \\
\hline M12** & & & & & 0.0000 & 0.0167 & 0.0250 & 0.0083 & 0.0169 & 0.0050 & 0.0169 & 0.0333 & 0.0583 & 0.0167 & 0.0083 \\
\hline M3 & & & & & & 0.0000 & 0.0417 & 0.0250 & 0.0169 & 0.0336 & 0.0169 & 0.0333 & 0.0333 & 0.0000 & 0.0250 \\
\hline M4 & & & & & & & 0.0000 & 0.0167 & 0.0254 & 0.0588 & 0.0254 & 0.0250 & 0.0500 & 0.0417 & 0.0167 \\
\hline M5 & & & & & & & & 0.0000 & 0.0254 & 0.0420 & 0.0254 & 0.0250 & 0.0500 & 0.0250 & 0.0167 \\
\hline M6 & & & & & & & & & 0.0000 & 0.0513 & 0.0172 & 0.0339 & 0.0424 & 0.0169 & 0.0085 \\
\hline M8 & & & & & & & & & & 0.0000 & 0.0051 & 0.0336 & 0.0420 & 0.0336 & 0.0059 \\
\hline M9 & & & & & & & & & & & 0.0000 & 0.0169 & 0.0424 & 0.0169 & 0.0085 \\
\hline M10 & & & & & & & & & & & & 0.0000 & 0.0417 & 0.0333 & 0.0250 \\
\hline M11 & & & & & & & & & & & & & 0.0000 & 0.0417 & 0.0500 \\
\hline M14 & & & & & & & & & & & & & & 0.0000 & 0.0250 \\
\hline M15 & & & & & & & & & & & & & & & 0.0000 \\
\hline
\end{tabular}

*Biomira mAbs; **CanAg mAbs; all other methods use Centocor/Fujirebio mAbs. In bold: discordance rates $>$ maximum percent discordance observed in method comparison using similar antibodies; in italic: comparison of methods using different mAbs.

Table 8 Results of ROC curve analysis for the different methods.

\begin{tabular}{|c|c|c|c|c|c|c|c|}
\hline Methods & $\begin{array}{l}\text { Manufacturer } \\
\text { cut-off value, } \\
\mathrm{U} / \mathrm{mL}\end{array}$ & $\begin{array}{l}\mathrm{n} \\
\text { "without } \\
\text { recurrence" }\end{array}$ & $\begin{array}{l}\mathrm{n} \\
\text { "with } \\
\text { recurrence" }\end{array}$ & $A \cup C$ & $\begin{array}{l}\text { Optimal } \\
\text { cut-off } \\
\text { value, } \\
\text { U/mL }\end{array}$ & $\mathrm{Se}, \% *$ & Sp, \%* \\
\hline M1 & 23.4 & 78 & 35 & 0.999 & 30.9 & 98.7 & 100.0 \\
\hline M2 & 38 & 84 & 35 & 0.998 & 39.0 & 97.6 & 100.0 \\
\hline M3 & 31.3 & 85 & 35 & 1.000 & 24.7 & 100.0 & 100.0 \\
\hline M4 & 25 & 83 & 35 & 1.000 & 28.9 & 98.8 & 100.0 \\
\hline M5 & 30 & 85 & 35 & 1.000 & 23.0 & 100.0 & 100.0 \\
\hline M6 & 30 & 79 & 35 & 1.000 & 25.1 & 100.0 & 100.0 \\
\hline M7 & 31.3 & 84 & 33 & 0.994 & 25.6 & 95.2 & 100.0 \\
\hline M8 & 30 & 76 & 35 & 0.994 & 24.7 & 96.1 & 100.0 \\
\hline M9 & 35 & 76 & 35 & 1.000 & 31.5 & 100.0 & 100.0 \\
\hline M10 & 30 & 69 & 35 & 0.999 & 25.0 & 97.1 & 100.0 \\
\hline M11 & 28 & 76 & 31 & 0.986 & 24.9 & 93.4 & 100.0 \\
\hline M12 & 35 & 68 & 35 & 1.000 & 34.3 & 98.5 & 100.0 \\
\hline M13 & 38.6 & 85 & 34 & 1.000 & 30.2 & 100.0 & 100.0 \\
\hline M14 & 32.4 & 85 & 35 & 1.000 & 22.9 & 98.8 & 100.0 \\
\hline M15 & 31.3 & 85 & 35 & 1.000 & 26.9 & 100.0 & 100.0 \\
\hline
\end{tabular}

AUC, area under ROC curve; Se, clinical sensitivity; Sp, clinical specificity; *values are overestimated since patients were not taken at random.

of standardizing tumour marker assays. Consequently, tumour marker results are known to be strongly dependent on the assay method, a concern that has complicated their acceptance for clinical use (18).

In the present work, we did not attempt to compare the analytical performance among different methods, as our focus was on evaluating the clinical relevance of the results. We chose to use individual patient sera only, and thus avoid bias due to artificial quality control preparations (19). In addition, all serum samples were tested by all 15 methods.

Taking AFSSAPS panel results as a whole, we found the rates of FP (elevated MUC-1/CA 15-3 in nonrecurring breast cancer patients), or FN (MUC-1/CA 15-3 results under cut-off in metastatic patients) to be consistent with those observed among classical biological parameters. A major conclusion of our work is that a uniform calculation by ROC curve analysis of the results obtained with patient groups of adequate size significantly reduces these discrepancies (20). As expected, sera from group 2 patients (recent first metastasis and moderate increases in MUC-1/CA 15-3), showed the highest $(3.1 \%)$ rate of FN results, depending on the analytical sensitivity of the techniques. Sera from this type of patient should be carefully studied by manufacturers to optimise the clinical sensitivity of the assay to enable early detection of metastases.

In the whole panel of sera, we observed considerable variations in the range of MUC-1/CA 15-3 concentrations. They varied from $395 \mathrm{U} / \mathrm{mL}$ to $18,315 \mathrm{U} / \mathrm{mL}$ for methods using Biomira Mabs, 247-930 U/mL for methods based on CanAg Mabs and from $275 \mathrm{U} / \mathrm{mL}$ to $18,983 \mathrm{U} / \mathrm{mL}$ for methods using Centocor/Fujirebio Mabs. This suggests that at least some methods are prone to hook effects in the presence of elevated MUC-1/CA 15-3, as was evidenced by three of us (JPB, NE and JMR) with the Kryptor method. 


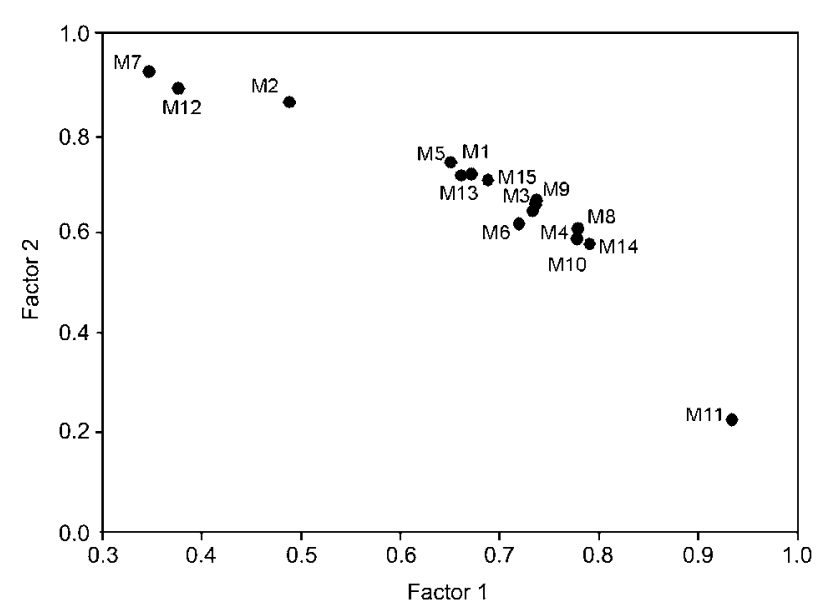

Figure 1 Results of principal component analysis. After Varimax rotation, methods are grouped according to their relative weight on the first and second component. M2, $\mathrm{M} 7$ and $\mathrm{M} 12=\mathrm{CanAg}$ antibodies, $\mathrm{M} 1$ and $\mathrm{M} 13=\mathrm{CA} 27.29$ (Biomira) mAbs, M3-M6, M8-M10, M11, M14-M15= Centocor/Fujirebio mAbs.

Discordance rates were low and homogenous among methods based on the same antibody type, but higher when comparing methods using different antibodies. This finding is illustrated by principal component analysis that grouped together methods based on Centocor/Fujirebio and Biomira mAbs. Epitopes recognised by DF3 $\mathrm{mAb}$ and antibodies against CA 27.29 (Biomira) differ by one amino acid only at their N-terminal side; the CA 27.29 being devoid of the Ala residue. Methods using CanAg Ma 552 antibody, which were displayed separately, recognise an epitope identical to the Biomira mAb, but with a Gly residue added on its C-terminal side. This probably results in a difference in the secondary structure of the Ma 552 epitope, slightly changing the specificity of that antibody.

Overall, this study highlights the pivotal importance of antibody type in the performance of MUC-1/CA 15-3 immunoassays: principal component analysis showed that it was the strongest determinant of assay results, weighing more than the immunoassay system used, the method category, or the type of endpoint signal measured.

\section{Conflicts of interest}

None.

\section{Acknowledgements}

The authors acknowledge Drs. Jean-Louis Floiras (Centre René-Huguenin, Saint-Cloud), Alain Pecking (Centre RenéHuguenin, Saint-Cloud) and Florence Dalenc (Institut Claudius Regaud, Toulouse) for expert clinical discussions. We acknowledge Béatrice Boucher, Agence Française de Sécurité Sanitaire des Produits de Santé, Saint-Denis, for her technical participation. We also acknowledge the biologists who performed some of the assays of the serum panel:
Dr Elmer, Laboratoire d' Eylau, Paris, Pr Debuire, hôpital Paul Brousse, Villejuif, Dr Troalen, Institut Gustave Roussy, Villejuif, Dr Lacroix, Laboratoire Pasteur-Cerba, Cergy-Pontoise, Dr Fouqueray, hôpital Tenon, Paris, Dr Burnat, hôpital d' Instruction des Armées Bégin, Saint-Mandé, Dr Maisonneuve, hôpital Robert Ballanger, Aulnay-sous-Bois, Dr Ramirez, hôpital d' Instruction des Armées Val de Grâce, Paris, Dr Poupon, hôpital Simone Veil, Eaubonne. The authors gratefully acknowledge Ms. Linda L. Fox assistance for reading the English text and making suggestions.

\section{References}

1. Taylor-Papadimitriou J, Burchell J, Miles DW, Dalziel M. MUC1 and cancer. Biochem Biophys Acta 1999;1455: 301-13.

2. Hanisch FG, Muller S. MUC1: the polymorphic appearance of a human mucin. Glycobiology 2000;10:43949.

3. Basuyau JP, Blanc-Vincent MP, Bidart JM, Daver A, Deneux L, Eche N, et al. Standards, Options et Recommendations (SOR): marqueurs tumoraux sériques du cancer du sein. Bull Cancer 2000;87:723-37.

4. Hilkens J, Kroenzen V, Bonfrer JM, Bruning PF, Hilgers J, Van Eijkeren A. A sandwich radioimmunoassay for a new antigen (MAM6) present in the sera of patient with metastazied carcinomas. In: Peeters $\mathrm{H}$, editor. Protides of the biological fluids. Oxford: Pergamon Press 1984; $651-3$.

5. Kufe D, Inghirami G, Abe M, Hayes D, Justi-Wheeler H, Schlom J. Differential reactivity of a novel monoclonal antibody (DF3) with malignant versus benign breast tumors. Hybridoma 1984;3:223-32.

6. Price MR, Hudecz F, O'Sullivan C, Baldwin RW, Edwards PM, Tendler SJ. Immunological and structural features of the protein core of human polymorphic epithelial mucin. Mol Immunology 1990;27:795-802.

7. Ceriani RL, Peterson JA, Blank EW, Lamport DT. Epitope expression on the breast epithelial mucin. Breast Cancer Res Treat 1992;24:103-13.

8. Briggs S, Price MR, Tendler SJ. Fine specificity of antibody recognition of carcinoma-associated epithelial mucins: antibody binding to synthetic peptide epitopes. Eur J Cancer 1993;29:230-7.

9. Price MR, Rye PD, Petrakou E, Murray A, Brady K, Imai $S$, et al. Summary report on the ISOBM TD-4 workshop: analysis of 56 monoclonal antibodies against the MUC1 mucin. Tumor Biology 1998;19(Suppl 1):1-20.

10. Cohen R, Zucchelli GC, Fraysse M, Pilo A, Rigault MY, Grillet S, et al. Oncocheck: an international external quality assessment scheme for immunoassays of tumor markers. Nucl Med Biol 1994;21:483-93.

11. Slev RS, Rawlins ML, Roberts WL. Performance characteristics of seven automated CA 15-3 assays. Am J Clin Pathol 2006;125:752-7.

12. Stern P, Bartos V, Vavrova J, Bezdickova D, Pechova M, Uhrova $\mathrm{J}$, et al. Comparability of eight immunoassay procedures for the determination of CA 15-3 related markers. Clin Chem Lab Med 2003;41:1087-94.

13. Agence Française de Sécurité Sanitaire des Produits de Santé (AFSSAPS). Rapport du contrôle du marché des DMDIV de MUC-1/CA 15-3. http://agmed.sante.gouv.fr/ $\mathrm{htm} / 10 / \mathrm{dm} / \mathrm{sdm} /$ rapport_controle_dmdiv_muc.pdf.

14. Fédération Nationale des Centres de Lutte Contre Le Cancer. Standards, Options et Recommandations: cancers du sein infiltrants non métastatiques, v.2 revised, Montrouge: John Libbey Eurotext, 2001:369 pp. 
15. Stenman UH. Immunoassay standardization: is it possible, who is responsible, who is capable? Clin Chem 2001;47:815-20.

16. Hubl W, Demant T, Albrecht S, Bewarder N, Grunow G, Keller, et al. A multi-center quality control study of different CA 15-3 immunoassays. Clin Lab 2005;51:641-5.

17. Van Dalen A. Analytical requirements and standardization of CA 15-3. Scand J Clin Lab Invest 1995;221(Suppl): 102-4.

18. Hilgers J, Von Mensdorff-Pouilly S, Verstraeten AA,
Kenemans P. Quantitation of polymorphic epithelial mucin: a challenge for biochemists and immunologists. Scand J Clin Lab Invest 1995;55(Suppl 221):81-6.

19. Klee G. MUC1 gene-derived glycoprotein assays for monitoring breast cancer (CA 15-3, CA 27-29, BR). Are they measuring the same antigen. Arch Pathol Lab Med 2004;128:1131-5.

20. Zweig $\mathrm{MH}$, Campbell G. Receiver-operating characteristic (ROC) plots: a fundamental evaluation tool in clinical medicine. Clin Chem 1993;39:561-77. 